\title{
Three-stage Kinetic Model for Self-decaying of Defects in Brittle Solids
}

\author{
V.I. Teslenko*, O.L. Kapitanchuk ${ }^{\dagger}$ \\ Bogolyubov Institute for Theoretical Physics, National Academy of Sciences of Ukraine, \\ 14-b, Metrolohichna St., 03680 Kyiv, Ukraine
}

(Received 04 August 2020; revised manuscript received 17 December 2020; published online 25 December 2020)

\begin{abstract}
Based on the density matrix method for a general nonequilibrium system consisted of a number of fluctuating in energy phonon-dressed states weakly coupled to the equilibrium environment, and using the concept of self-decaying defect states in terms of the three-stage framework for the defect dynamics, the applied cumulative stress distributions of a failure probability for the non-stationary population of peak amplitudes of intermediate state of the three-state decaying nonequilibrium system are found. It is shown that the theoretical cumulative distribution determined for this state in terms of the respective solution of transcendent equation for the maximum of population is in direct correspondence with the damage probability of the whole system and therefore should be in agreement with the experimental cumulative distribution of the irreversible failure of the system observed on flexural testing of the brittle solids. In the proposed formalism, it is established that a-plane sapphire is advanced in its brittle performance. As such it is concluded that a- $\mathrm{Al}_{2} \mathrm{O}_{3}$ has not only far more strength, but reveals a noticeably higher competitive advantage as compared to CVD-ZnSe. This conclusion agrees well with the corresponding experimental observations provided in respective IR-transmitting window materials for the a- $\mathrm{Al}_{2} \mathrm{O}_{3}$ and CVD-ZnSe.
\end{abstract}

Keywords: Brittle solids, Self-decaying defect states, System failure, a- $\mathrm{Al}_{2} \mathrm{O}_{3}$ performance.

DOI: 10.21272/jnep.12(6).06017

PACS numbers: 05.70.Ln, 31.70.Hq, 42.60.Lh

\section{INTRODUCTION}

All solids, crystalline or amorphous, are not perfect in structure and have a certain amount of various defects. The types of these are numerous including vacancies and interstitial atoms (point defects), dislocations (line defects), stacking faults (planar defects), and voids (volume defects), to name a few. In fact, in any solid, a defect of every possible type has a non-zero concentration. Therefore, in order to perform successfully, a solid must tolerate the low concentration of defects by being endowed with the capability for its self-decaying irrespective of the type. This requires considering the defects in solids that as not the static macroscopic objects like permanent dislocations or structural imperfections, whose deleterious behavior scales linearly with the applied stress, but essentially as the dynamic microscopic objects like local or extended lattice deformations, which can depend on normal vibrational excitations (phonons) and whose behavior scales nonlinearly (exponentially) with the stress. Moreover, in a- $\mathrm{Al}_{2} \mathrm{O}_{3}$ brittle solid, such a nonlinear behavior can at the same time be stochastic, revealing in itself an irreversible fracture of a tested solid at a different random value of a slow ramped stress without any significant plastic deformation before one-off failure occurs $[1,2]$.

At the same vein, this means that to make a rigorous consideration of defect dynamics in solids, one needs to start from a microscopic framework of a defect-state space defined for a finite state nonequilibrium system coupled weakly to an infinite state equilibrium environment, and then to describe the defect evolution as the random movement of distorted groups of the system's atoms between the several preferable configurations (the so-called defect states) on the background of

\footnotetext{
*vtes@bitp.kiev.ua

† alkapt@ukr.net
}

harmonic vibrations of environmental atoms. Such raison d'être recognized long ago is formulated consistently in [3], where the defects are associated with elastic distortions of nonequilibrium atomic configurations interacting with normal vibrations of the bulk atoms which form the reservoir of thermally equilibrated phonons. Because the defect-phonon interaction is introduced microscopically, this not only allows to nonperturbatively define the renormalized defect states dressed in virtual phonons, but also makes it possible to calculate the rate constants of relaxation transitions between the dressed states. In this framework, relaxation processes are re-assumed to be induced by the defect-phonon interaction. As the latter after renormalization is made small enough to be taken as a perturbation, this justifies the use of lowest-order-perturbation approximation (Born approximation) and provides a basis for the explanation of general regularities in the dynamics of various defects in ceramics as well [4].

In the similar framework developed in [4] the structural defects are associated with some kinds of disorder in nearly periodic amorphous lattices, too, provided that the emerged defect states interacting with each other and with lattice phonons are properly (or, at least, consistently) defined. Moreover, the microscopic nature of these defects can be very different, from the disordered pattern of molecular bonds in the lattice (like loose atoms or dangling bonds) having mainly two alternative positions in space (the two-state defect) separated by a particular potential barrier, to the extended structural configurations of compact disordered regions encompassing hundreds of molecular units and forming the multi-state defects. Analogously, the structure and properties of $\mathrm{Al}_{2} \mathrm{O}_{3}$ associated with the deformation of the lattice in the vicinity of the vacancy can be described 
within the framework described in [5].

In the present paper, based in part on our previous work [3], we aim at developing the three-stage selfdecaying model able to describe the irreversible dynamics of defects in brittle solids, from a microscopic standpoint. Our approach is formulated in the framework of a microscopic defect-state space for a nonequilibrium nonstationary system coupled to an equilibrium steadystate environment. But in contrast to [3], we allow the system's eigenenergy levels with random fluctuations around the stochastic average positions with mean amplitudes and frequencies. This allows to use the formalism of calculating the rate constants of transitions between the fluctuating energy levels developed in [4].

The paper is organized as follows. In Section 2 we propose the theoretical model for describing the dynamics of a- $\mathrm{Al}_{2} \mathrm{O}_{3}$ defects in a nonequilibrium system consisted of a number of fluctuating in energy phonondressed states weakly coupled to the environment that induces relaxation transitions between them in the one-defect approximation. In Section 3, for the case of a single transient failure-prone state, we reduce this model to a three-stage chain of transitions, for which the solution for the time-dependent population of that state is obtained in an analytical form in Section 2. This allows to find the stress dependence of the maximum of the population that determines the cumulative failure distribution. The theoretical results are compared with experimental data on flexural testing of aplane sapphire in Section 3. Finally, in Section 4, the consequences thereof are concluded.

\section{THEORETICAL MODEL}

There are different scales for description of the dynamics of defects in a nonequilibrium system. At each scale, one aims to model the temporal behavior of defects in the corresponding time and state spaces characteristic of the required description level. As such it is commonly assumed that, despite the different types of defects may occur in the system in all possible occupation numbers, there is the mean field approximation that replaces an actual surrounding of a defect by a locally averaged number of neighboring defects, so that solely a single defect of the most relevant type will influence one and only one set of observations [3]. As a consequence, the many-defect state can be specified in terms of the occupation numbers (populations of defect states) associated with the small elementary cells in the system defined at the level of a single-defect state-space scale.

In general, one can indicate three levels of description at which the plausible models for defects may be defined most accurately. These are microscopic level, mesoscopic level, and macroscopic level. At the macroscopic level, defects are associated with some macroscopic particles such as specific atomic and molecular structures, whose concentrations can vary with time and behavior of which obeys the generalized continuity equation in the reactor. However, if to use both the low concentration limit and an activated state representation for a single defect species, then equation of continuous diffusion of a defect can easily be reduced to the master equation for its thermally activated Arrheniustype transitions between failure-tolerant and failure- prone system states [4]. Analogous reduction of the dynamics of defects to the master equation for their between-state transitions is also appropriate for the mesoscopic level, at which the defects are associated with the mesoscale faults such as localized cracks, whose density exponentially depends on the stress.

However, at the microscopic level, one cannot characterize the defects so specifically as in cases of macroscopic and mesoscopic level descriptions. The matter of fact is that microscopic defects, being numerous, but small in size, are hidden from direct observations and even undetectable in a brittle solid for causing no harm to it. Therefore, in order to maintain the defect concentration at a no damage level avoiding brittle failure of the solid, there should be a particular mechanism that balances the process of thermal activation of defects with their spontaneous self-decaying by matching a difference between numbers of stationary appearing and disappearing defects at the subthreshold level. Moreover, in this case, the defects will be associated with the stochastically fluctuating thermally-excited states of a nonequilibrium quantum system weakly coupled with its equilibrium environment [5, 6]. Remarkably, the kinetic equation for populations of defect states, found as a result of stochastic averaging of the microscopic Liouville-von Neumann evolution equation for the density matrix of the whole system "system + environment + weak interaction" over fast fluctuations in the states' energies, being generally nonlinear and containing also states' coherences, can nevertheless be reduced to a linear equation of the population balance only [4].

In general, the microscopic master equation is too complicated to treat it precisely. Therefore, to try to integrate it analytically we need simplifying approximations dealing with averaging out those irrelevant degrees of freedom, precise knowledge of which is out of the question. For doing this, according to Ref. [3], the sequence of consecutive averaging approximations can be proposed as follows. These approximations are: first, partial averaging over the Bose-distributed normal vibrations (phonons) in a thermally equilibrium environment by eliminating them from explicit consideration; then, neglecting the fast decaying off-diagonal elements of the nonequilibrium density matrix (states' coherences) and retaining only its ensemble-averaged diagonal elements (populations of system states); further, averaging over the random fluctuations in microscopic energies given the distributions of their mean stochastic amplitude and frequency parameters; and, finally, modularizing the description of the temporal behavior of the locally equilibrium state populations by using appropriate steady-state approximations (for more detail, see, e.g. [4]). The final result of the above approximations is the reduced master equation.

Following [7], the resulting kinetic model for the process of self-decaying of defects in a brittle solid in the one-defect approximation is represented in the form of a three-stage four-state scheme

$$
|I\rangle \underset{r}{\stackrel{f_{\sigma}}{\longleftrightarrow}}|F\rangle \underset{\longleftarrow_{\beta}}{\stackrel{\alpha}{\longrightarrow}}|D\rangle \stackrel{\gamma}{\longrightarrow} .
$$

Here $\mid I=\{i\}_{1, \ldots, N_{\sigma} \equiv n_{\sigma}^{-1}}>$ is the initial failure-tolerant 
state being the aggregated state of $N$ equipopulated (degenerate) thermally activated states at equal energy $E_{I}$ with the initial equilibrium population, $|F\rangle$ is the transient failure-prone state at energy $E_{F}, \mid D>$ is the decay failure-tolerant state at energy $E_{D}$, and $f_{\sigma}, r, \alpha, \beta, \gamma$ are the rate constants for between-state transitions directed with respective arrows in (1). In this scheme, the activated-state degeneracy, being a function of $T$ and applied stress $\sigma$, scales with these as

$$
\begin{aligned}
& N_{\sigma} \equiv n_{\sigma}^{-1}=\exp \left[\left(E_{I}-\sigma \Omega\right) / k_{B} T\right] \times \\
& \times\left\{1+\exp \left[-\left(E_{I}-\sigma \Omega\right) / k_{B} T\right]+\right. \\
& \left.+\exp \left(-E_{F} / k_{B} T\right)+\exp \left(-E_{D} / k_{B} T\right)\right\}
\end{aligned}
$$

Here, $\Omega$ is the volume of representative volume of a one-defect cell of the system whose states of applied stress are uniformly distributed in the cell with activation energies being much larger than the thermal energy

$$
E_{I}>E_{F}>E_{D}>k_{B} T
$$

At not very high stresses, the expression in the figure brackets of (2) is close to unity. Therefore, the steadystate stress in the one-defect cell is determined by the condition of chemical potential equilibrium

$$
\sigma \Omega-k_{B} T \ln \left(n_{\sigma}^{-1} / n_{0}^{-1}\right)=0,
$$

where $n_{0}=\exp \left[-\left(E_{I} / k_{B} T\right)\right]$ is the activated-state degeneracy density at zero stress. However, condition (4) is well appropriate for a continuous distribution of defect states ceases to hold at higher stresses in the one-defect approximation conditioned by a discrete degeneracy relation $N_{0} \gg N_{\sigma} \geq 1$. Therefore, in this case one must use the more general expression (2) which reproduces both the limit of zero stress and the limit of infinite stress leading to $N_{0} \gg 1$ and $N_{\sigma \rightarrow \infty}=1$, respectively.

The kinetic model (1) comprises the problem of a continuous-time discrete-state Markov chain. Given the initial conditions, it can be solved exactly for the timedependent populations of states by using standard numerical methods for solving systems of first order linear differential equations [3].

Hence, it is appropriate to restrict further consideration of defect evolution to only the one failure-prone state $|F\rangle$, staying with a defect which appears for the system to be a single cause for failure. However, this state is a transient one. So, transiting a defect to the pre-decay state $\mid D>$ makes the system failure-tolerant and hence creates the possibility for ultimate self-decaying of it to the ground defect state. The resulting system of master equations in the case (1) for the respective state population $p_{I, F, D}(t)$ takes the form of the system of three kinetic equations

$$
\left\{\begin{array}{l}
\dot{p}_{D}(t)=-(\beta+\gamma) p_{D}(t)+\alpha p_{F}(t) \\
\dot{p}_{F}(t)=\beta p_{D}(t)-(r+\alpha) p_{F}(t)+f_{\sigma} p_{I}(t) \\
\dot{p}_{I}(t)=r p_{F}(t)-f_{\sigma} p_{I}(t)
\end{array}\right.
$$

However, even for the given initial conditions, the analytical solution of system (5) remains still complicated. Nevertheless, we can use an exact analytical solution to the similar simpler system obtained in [3] and find an approximate analytical solution for population $p_{F}^{(\sigma)}(t) \equiv p_{F}(t ; \sigma)$ of failure-prone state $|F\rangle$ (the stationary level that is always small at all $\sigma$ ). By so doing, we can obtain

$$
p_{F}(t)=f n_{\sigma} \sum_{j} \frac{\beta+\gamma-\lambda_{j}}{\prod_{j \neq j^{\prime}}\left(\lambda_{j^{\prime}}-\lambda_{j}\right)} \exp \left(-\lambda_{j} t\right) .
$$

Here, the exponents $\lambda_{j^{\prime} \neq j=1,2,3}$ under summation and product signs are the system eigenvalues which are associated with the non-negative Debye relaxation rates obeying the characteristic equation

$$
\begin{aligned}
& \lambda^{3}-\lambda^{2}\left(f n_{\sigma}+r+\alpha+\beta+\gamma\right)+ \\
& +\lambda\left[\left(f n_{\sigma}+r\right)(\beta+\gamma)+\alpha\left(f n_{\sigma}+r\right)\right]-f n_{\sigma} \alpha \gamma=0
\end{aligned}
$$

Given the simple initial conditions, analytical solution (6) coincides with the analogous solution of [3] at $N_{\sigma}=1$. Moreover, Eq. (7) corresponds to the third order equation

$$
\begin{aligned}
& \dddot{p}_{F}(t)+\left(f n_{\sigma}+r+\alpha+\beta+\gamma\right) \ddot{p}_{F}(t)+ \\
& +\left[\left(f n_{\sigma}+r\right)(\beta+\gamma)+\alpha\left(f n_{\sigma}+r\right)\right] \dot{p}_{F}(t)+f n_{\sigma} \alpha \gamma p_{F}(t)=0
\end{aligned}
$$

This equation represents an exponential temporal rise and the decay pattern that consists of the failure relaxation modes. This mode adds its particular contribution, differing in eigenvalue (7), as well as amplitude and sign (8). As expected, the time dependence of $p_{F}^{(\sigma)}(t)$ is transient and shows an increase, peak and decline without oscillations to the equilibrium as $t \rightarrow \infty$. This allows us to find the maximum of the population

$$
\bar{p}_{F}^{(\sigma)}=p_{F}^{(\sigma)}\left(\bar{t}_{F}^{(\sigma)}\right)
$$

at the peak time moment being a nontrivial solution of the transcendent equation

$$
\dot{p}_{F}^{(\sigma)}(t)=0 .
$$

Since the maximum failure probability is associated with just a maximum of population (6) of failure-prone state $\bar{p}_{F}^{(\sigma)}(t) \equiv \bar{p}_{F}(t ; \sigma)$, expression (9) represents the stress dependence of failure cumulative distribution function of the system. Populations $p_{D, I}^{(\sigma)}(t)$ of other states can be obtained similarly (cf. [3]) and are not presented here for reasons of space.

In general, there is no analytical solution to the problem (6)-(10). Therefore, in the next section we use a numerical method to simulate the behavior of failure cumulative distribution based on available experimental data for brittle ceramic materials.

\section{COMPARISON WITH EXPERIMENT}

In the previous section, we propose a three-stage kinetic scheme (1) to model the self-decaying of defects 
in brittle solids under the applied stress. In the onedefect approximation, we consider the time behavior of four defect states, that is, the ground state, decay state $|D\rangle$, failure-prone state $|F\rangle$, and aggregated activated state $|I\rangle$, populations of which obey the system of kinetic equations (3). We assume that in this system the initially populated is only activated state and the responsibility for failure lies with only failure-prone state. In approximations of the high degeneracy of activated state and the large energy of activated, failureprone and decay states as compared to the energy of the ground state, we obtain the stress dependence for the failure cumulative distribution function (9) associated with the maximum $\bar{p}_{F}^{(\sigma)}$ of population $p_{F}^{(\sigma)}(t)$ of failure-prone state $|F\rangle$.

In order to make a comparison of theoretical cumulative distribution $\bar{p}_{F}^{(\sigma)}$ (9) with experimental data we must select a structural material that is brittle and hence prone to fracture. In this regard, the most appropriate materials are infrared window materials, such as CVD ZnSe and sapphire, widely used in automobile, aerospace and space structures and electronic devices [8-10]. These materials are tough to resist fracture under sudden impact, but produce a brittle fracture response with little to no deformation. Therefore, it is natural and interesting to test those materials in the conditions that could imitate the effects of flexural stresses on deleterious strains in them. Such a testing has actually been carried out by Klein in a number of his papers e.g. $[6,8,10]$. It was shown that the Weibull statistics of brittle ceramics do not always well describe the experimental cumulative distributions and should be endowed with additional parameters to better fit the data [11]. On the other hand, these distributions can successfully be described by the use of statistics of decaying defect states in the framework of three-stage kinetic model coincident with (9) and considered in detail in [3]. Consequently, we may use here the results obtained in [3] to illustrate that, in the one-defect approximation, the applicability of a kinetic model considering only three decaying states, and one absorbing state to the description of experimental data can serve as the evidence for thermal creation and spontaneous self-decaying of defects in brittle solids. The very similar effects of thermal creation and spontaneous decaying of defects have also been observed in polyethylene crystals.

Usually, different brittle materials have different margins of safety with respect to flexural stress [8-10]. Therefore, using two different strength parameters and two different shape parameters to describe the dependence of measured cumulative failure distributions on the applied stress in terms of the five parameter bimodal Weibull distribution model [10] is almost insufficient for the possibility of a significant stress dependence of all those parameters. On the other hand, the use of five parameter rate constant space of the kinetic model (1) in the one-defect approximation (cf. [3]) instead of two-strengths-two-shapes-one-weight parameter space of bimodal Weibull model noted above appears to be fully independent of the stress scale. The main reason for this is that, in a thermodynamic model (5), the stress is assumed to change only degeneracy of aggregated activated state $f_{\sigma} \equiv f n_{\sigma}$, but not influence microscopic rate constants of transitions between different states in the kinetic model (1).

It is known that the system (6) with $M+1$ states generally has $M(M+1) / 2$ rate constants with only $2 M-1$ of them be linearly independent in the leadingorder approximation. As the set of microscopic rate constants is scale independent, it can therefore be considered as a complete set of independent parameters in a rate constant space of scheme (1). Moreover, we can introduce:

$$
\bar{f}_{\sigma} \equiv f_{\sigma} / w ; \bar{r} \equiv r / w ; \bar{\beta} \equiv \beta / w ; \bar{\alpha} \equiv \alpha / w ; \bar{\gamma} \equiv \gamma / w
$$

The set of transformed parameters becomes dimensionless, with $w$ being the failure rate constant corresponding to the median of cumulative failure distribution function $\bar{p}_{F}^{(\sigma)}$ (9) measured at the stress $\sigma=\sigma_{m s}$, where $\sigma_{m s}$ is the median strength characteristic of the material.
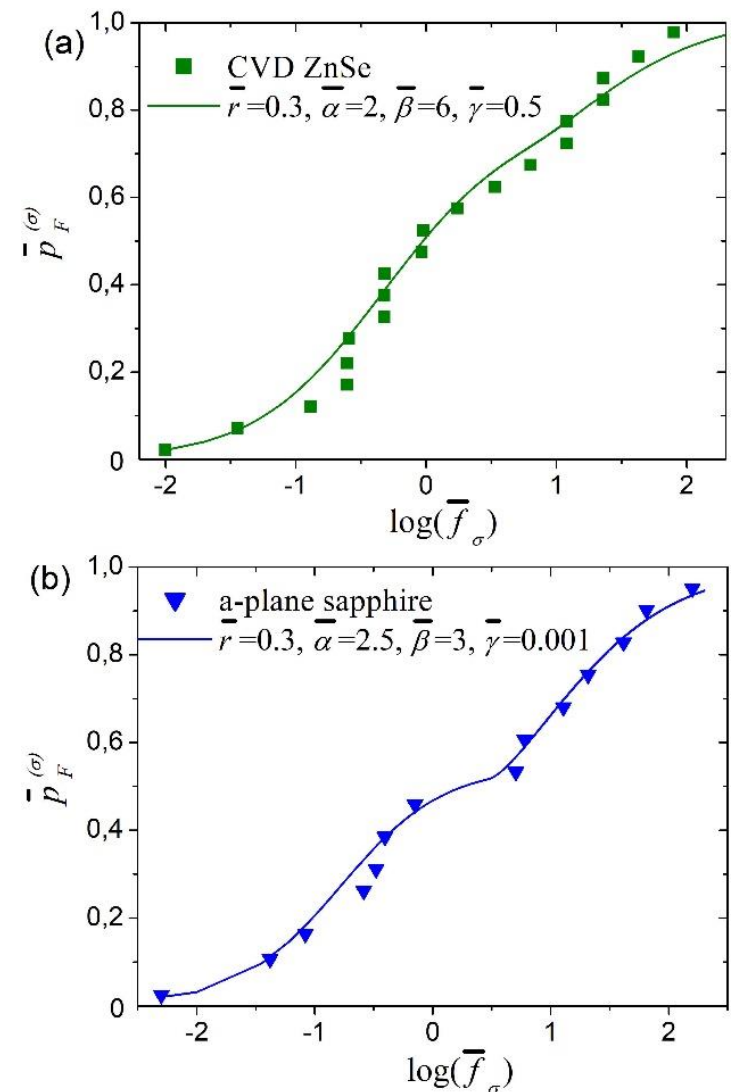

Fig. 1 - The cumulative failure distribution $p_{F}^{(\sigma)}$ (curve) simulated with (6)-(10) for (a) CVD ZnSe and (b) sapphire with the rate constant parameters (given in the inset in dimensionless units) as a function of dimensionless reduced stress logarithmically scaled as $\left(\sigma-\sigma_{m s}\right) \Omega /\left(k_{B} T \ln 10\right)=\log \left(\bar{f}_{\sigma}\right)(12)$. The experimental data for both materials (squares and triangles) are adapted from [8]

This allows to give a description of experimental data on the flexural load testing of the two different brittle solids, that is, CVD ZnSe and sapphire, in a unified manner. In such a description, the transformed failure rate constant $\bar{f}_{\sigma}=\bar{f} n_{\sigma}$ is considered as a running pa- 
rameter that can change with stress due to (2) in the wide limits by satisfying the relation

$$
\sigma-\sigma_{m s}=\left(k_{B} T / \Omega\right) \ln \left(d \bar{f}_{\sigma}\right),
$$

whereas the other transformed rate constants are regarded as the adjusting parameters specific to a brittle solid examined with $\sigma_{m s} \equiv\left(k_{B} T / \Omega\right) \ln \left(1 / \bar{f}_{\sigma}\right)$ being a nuisance parameter characteristic of its median strength.

Thus, experimental data for CVD ZnSe and sapphire, adapted from [8], can be compared with the corresponding cumulative failure distribution functions (9) numerically simulated using the equations (6)-(10). The result of this comparison is depicted in Fig. 1. As it is seen, adjusting the dimensionless rate constant parameters (9) with accuracy to the arbitrary failure rate constant $\bar{f}_{\sigma}$ that is generally unknown provides a good agreement between the theory and the experiment. Moreover, both the nearly unimodal cumulative failure distribution in Fig. 1a and the clearly bimodal cumulative failure distribution in Fig. 1b are described equally well. This means that the unification of rate constant space for the description of evolution of self-decaying defect states in a simple three-stage kinetic model (1) can form a helpful framework for insights into the stress behavior of brittle solids whose cumulative failure distributions are sigmoidal with different steepness slopes and modality shapes, but detailed information on the median strengths of which is hardly accessible or almost inaccessible for cost or technical reasons.

The first reason is associated with the relatively simple problem of unifying the description of cumulative distributions far distanced in their strengths on the stress scale. To represent these distributions on a single stress scale, that problem is approached with numerical simulations of equations (6)-(10) on a reduced rate constant space. This allows to provide a comparison of experimental failure cumulative distributions and theoretical ones without taking into account the strengths of the materials and without specifying their failure rate constants. A result of a comparison is shown in Fig. 1.

The second reason is related to the complicated problem: resolving the sensitivity of the response of different materials to the stress loading. Obtaining the density distribution functions from the cumulative distribution functions $\bar{p}_{F}^{(\sigma)}$ as $d\left\{\bar{p}_{F}^{(\sigma)}\left[\ln \left(f_{\sigma}\right)\right]\right\} / d\left[\ln \left(f_{\sigma}\right)\right]$ and plotting them in Fig. 2 as two curves labeled as (1) and (2), respectively, we see that they are indeed very different. Thus, the highest slope steepness of cumulative distribution functions manifested in the largest maximum of the density distribution function curves appears to be bigger in curve (1) than in curve (2) of Fig. 2, while the modality feature is more apparent in curve (2) than in curve (1).

The explanation of this effect is that a-plane sapphire is more advanced in its performance than ZnSe and hence shows the far more strength and a higher competitive advantage regarding to it (cf. [3]). This fully corresponds to the well-known maximizing performance while minimizing sensitivity-to-failure objective very typical of high-energy systems, such as missile window materials [6]. On the other hand, this implies that, given the tolerances with respect to the cumulative response of maximum population $\bar{p}_{F}^{(\sigma)}$ of failure-prone state $|F\rangle$ on the limiting stimulus actions, such as flexural or tensile stress, the brittle material with the larger margin of safety in the log of stimulus rate constant space, such as a-plane sapphire $\left(\mathrm{a}-\mathrm{Al}_{2} \mathrm{O}_{3}\right)$ with density distribution curve (2) in Fig. 2, i.e., with the lower sensitivity (the slope of the cumulative stimulus response curve in Fig. 1b) exhibits lower sensibility to failure and therefore a higher competitive advantage as compared to the other brittle material, say $\mathrm{ZnSe}$, with the smaller margin of safety on the density distribution curve (1) in Fig. 2 and the higher slope of cumulative stimulus-response curve in Fig. 1a, if these materials are regarded as rivals or competitors [6].

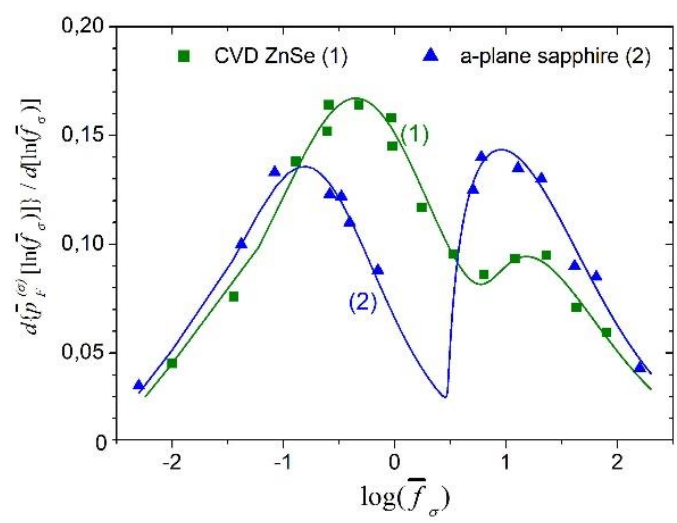

Fig. 2 - The cumulative failure distribution $p_{F}^{(\sigma)}$ (curve) simulated with (6)-(10) for (a) CVD ZnSe and (b) sapphire with the rate constant parameters (given in the inset in dimensionless units) as a function of dimensionless reduced stress logarithmically scaled as $\left(\sigma-\sigma_{m s}\right) \Omega /\left(k_{B} T \ln 10\right)=\log \left(\bar{f}_{\sigma}\right)(12)$. The experimental data for both materials (squares and triangles) are adapted from [8]

\section{CONCLUSIONS}

Based on the above results, we come to the conclusion that the concept of self-decaying of defect states in brittle solids can be considered as a consistent and physically well-substantiated framework helpful in understanding a variety of failure factors that influence the performance and competitiveness of different materials at various levels of fracture. Thus, among two IRtransmitting window materials analyzed in this work, the a-plane sapphire exhibits advanced performance revealing a higher competitive advantage regarding the CVD ZnSe, which has much lower strength (see Fig. 1) and moreover performs poorly compared to the a- $\mathrm{Al}_{2} \mathrm{O}_{3}$ (see Fig. 2). This conclusion agrees completely with the experimental observations provided in [8].

\section{ACKNOWLEDGEMENTS}

The present work was partially supported by the National Academy of Sciences of Ukraine (project No. 0116U002067). 


\section{REFERENCES}

1. T.L. Anderson, Fracture Mechanics - Fundamentals and Application, 3rd ed. (CRC Taylor \& Francis: Boca Raton: 2005).

2. R. Morrell, Fractography of Brittle Materials (National Physical Laboratory: Teddington: 1999).

3. O.L. Kapitanchuk, V.I. Teslenko, Mol. Cryst. Liqu. Cryst. 670 No 119 (2018).

4. O.L. Kapitanchuk, O.M. Marchenko, V.I. Teslenko, Chem. Phys. 472 No 249 (2016).
5. D.A. Kolesnikov, I.V. Sudzhanskaya, I.Yu. Goncharov, S.V. Lytovchenko, V.Yu. Novikov, E.A. Kudryavtsev, B.O. Mazilin, E.V. Krytsyna, V.M. Beresnev, O.V. Glukhov, J. Nano- Electron. Phys. 12 No 1, 01022 (2020).

6. C.A. Klein, Opt. Eng. 37, 2826 (1998).

7. O.L. Kapitanchuk, V.I. Teslenko, Phys. Chem. Solid State 20 No 3, 269 (2019)

8. C.A. Klein, Proc. SPIE 7504, 40K (2009).

9. C.A. Klein, J. App. Phys. 96, 3172 (2004)

10. C.A. Klein, Opt. Eng. 50, 023402 (2011).

\section{Три-стадійна кінетична модель для самозагасаючих дефектів в крихких твердих тілах}

\section{В.I. Тесленко, О.Л. Капітанчук}

Інститут теоретичної фізики ілені М.М. Боголюбова, Національна акаделія наук Украӥни, вул. Метрологічна 14-б, 03680 Київ, Украӥна

Ірунтуючись на методі матриці густини для загальної нерівноважної системи, яка розглядае певне число флуктуючих, одягнених фононами, станів, зв'язаних з рівноважним оточенням, та використовуючи самозагасаючі стани, за допомогою три-стадійного підходу до динаміки дефектів, знайдено кумулятивні розподіли ймовірностей руйнування в залежності від прикладеного напруження відносно піків амплітуд нестаціонарної заселеності проміжного стану системи, що загасає. Показано, що теоретичні кумулятивні розподіли, знайдені з розв'язку трансцендентного рівняння для амплітуд заселеностей проміжного стану, знаходяться у прямій відповідності з процесом необоротного руйнування всіеї системи і тому мають співпадати з експериментальними розподілами руйнування, які спостерігаються у тестах згинання крихких твердих тіл. У формалізмі, який пропонуеться, встановлено, що сапфір в а-площині відрізняеться перевагою у своїй якості щодо крихкості. При цьому зроблено висновок, що а- $\mathrm{Al}_{2} \mathrm{O}_{3}$ мае не тільки більшу стійку стабільність, а проявляє й помітно більш високу конкурентну перевагу відносно до CVD-ZnSe. Цей висновок добре узгоджуеться з експериментальними спостереженнями прозорих в IЧ-діапазоні відповідних матеріалів, як-то а- $\mathrm{Al}_{2} \mathrm{O}_{3}$ та CVD-ZnSe.

Ключові слова: Крихкі тверді тіла, Самозагасаючі стани, Відмова системи, Якість а- $\mathrm{Al}_{2} \mathrm{O}_{3}$. 